\title{
Clinical significance of lymphocytopenia in patients hospitalized with pneumonia caused by influenza virus
}

\author{
Valeria Bellelli, Gabriella d'Ettorre, Luigi Celani, Cristian Borrazzo, Giancarlo Ceccarelli and Mario Venditti (1)
}

Recently, lymphocytopenia has been evaluated as an independent biomarker of mortality in hospitalized patients diagnosed with community-acquired pneumonia (CAP) $[1,2]$. In these studies, patients recruited were affected by CAP due to different etiology, and no specific differences have been observed in viral and bacterial etiology distribution. On the other hand, in influenza virus infection, lymphocytopenia has been identified as a risk factor for bacterial superinfections [3], determining a worse prognosis. We would like to evaluate the potential role of lymphocytopenia as a prognostic factor in patients with pneumonia caused by influenza virus.

For this purpose, we performed a retrospective, observational study on patients hospitalized in a hospital in Rome with pneumonia due to influenza virus. Between January and April 2019, we observed 38 patients with either CAP (29 patients) or hospitalacquired pneumonia (9 patients) due to influenza virus (defined by the presence of fever, symptoms and signs of pneumonia syndrome, new onset of pulmonary infiltrates on chest X-rays or CT scans, and influenza virus detection on respiratory specimens). Consistent with already published data [4], the rate of nosocomial infections found was high and requires substantial improvement of early diagnosis and infection control strategies. Focusing on the lymphocyte count at the onset of infection, with the adoption of a previously reported cutoff value of 724 lymphocytes/ $\mu \mathrm{l}$ [2], 23 patients were considered as affected by lymphocytopenic influenza virus pneumonia (L-IP) and 15 by a non-lymphocytopenic influenza virus pneumonia (NL-IP). As shown in Table 1, in com- parison with NL-IP, patients with L-IP were more commonly affected by COPD $(p=0.046)$, they more frequently required admission to the intensive care unit $(p=0.002)$ and invasive mechanical ventilation $(p=0.031)$ and presented a higher SOFA score at the time of diagnosis $(p=0.004)$; they also experienced more frequently secondary bacterial and fungal pulmonary superinfections. As shown in Table 1, secondary bacterial pathogens were often multiple resistant nosocomial organisms as carbapenem-resistant Acinetobacter baumannnii and Corynebacterium striatum and methicillin-resistant Staphylococcus aureus. Notably, fungal pathogens were represented not only by Aspergillus fumigatus but also by Pneumocystis jiro$v e c i$. If the former association is well known, to our knowledge, only one case report has been published on $P$. jiroveci superinfection in an immunocompetent host affected by influenza virus [5]. Analyzing only the influenza CAP patients, the results were similar: SOFA score at the time of diagnosis was higher in patients with L-IP $(p=0.013)$ and they experienced more frequently respiratory failure requiring oxygen support $(p<0.001)$ and IMV $(p=0.045)$ (Table 1$)$. Moreover, all the episodes of superinfection were experienced by lymphocytopenic patients.

In our experience, although we evaluated a small sample, a more severe course of a disease might be expected in episodes of L-IP. Under these circumstances, even otherwise immunocompetent patients seem to be at increased risk for opportunistic pulmonary superinfections. Based on the abovementioned, L-IP would require close clinical monitoring for these potentially fatal infectious complications possibly including anti-Aspergillus prophylaxis.

* Correspondence: mario.venditti@uniroma1.it

Department of Public Health and Infectious Diseases, University of Rome Sapienza, Viale del Policlinico 155, Rome, Italy 
Table 1 Baseline characteristics, severity, and microbiology of pulmonary superinfections and outcomes

\begin{tabular}{|c|c|c|c|c|c|c|}
\hline \multirow[t]{2}{*}{ Characteristics } & \multicolumn{3}{|c|}{ Total influenza pneumonia (38) } & \multicolumn{3}{|l|}{ Influenza CAP (29) } \\
\hline & $\begin{array}{l}<724 \text { lymphocytes } / \mu \text { l } \\
(n=23)\end{array}$ & $\begin{array}{l}\text { > } 724 \text { lymphocytes } / \mu \text { l } \\
(n=15)\end{array}$ & $\begin{array}{l}p \\
\text { value }^{*}\end{array}$ & $\begin{array}{l}<724 \text { lymphocytes } / \mu \text { l } \\
(n=20)\end{array}$ & $\begin{array}{l}>724 \text { lymphocytes/ } \mu \text { l } \\
(n=9)\end{array}$ & $\begin{array}{l}p \\
\text { value }\end{array}$ \\
\hline Age, years & $71(57-80)$ & $76(66-80)$ & 0.681 & $70(58-78)$ & $78(66-80)$ & 0.681 \\
\hline Male sex & $6(26 \%)$ & $8(53 \%)$ & 0.291 & $4(20 \%)$ & $4(44 \%)$ & 0.188 \\
\hline $\mathrm{N} / \mathrm{L}$ ratio & $14.8(9.4-19.3)$ & $4.9(1.6-8.6)$ & $\begin{array}{l}< \\
0.001\end{array}$ & $15.5(12.9-30.6)$ & $6.8(3.3-7.8)$ & $\begin{array}{l}< \\
0.001\end{array}$ \\
\hline Current smoking & $17(74 \%)$ & $6(40 \%)$ & 0.098 & $15(75 \%)$ & $3(33 \%)$ & 0.034 \\
\hline Alcohol abuse & $1(4 \%)$ & $0(0 \%)$ & 0.413 & $1(5 \%)$ & $0(0 \%)$ & 0.502 \\
\hline Corticosteroid & $1(4 \%)$ & $1(7 \%)$ & 0.754 & $1(5 \%)$ & $1(11 \%)$ & 0.754 \\
\hline Influenza virus CAP & $20(87 \%)$ & $9(60 \%)$ & 0.368 & - & - & - \\
\hline Influenza virus HAP & $3(13 \%)$ & $6(40 \%)$ & 0.656 & - & - & - \\
\hline \multicolumn{7}{|l|}{ Comorbidity } \\
\hline Cardiovascolar & $18(78 \%)$ & $10(67 \%)$ & 0.428 & $16(80 \%)$ & $5(56 \%)$ & 0.188 \\
\hline Neurologic & $7(30 \%)$ & $6(40 \%)$ & 0.543 & $6(30 \%)$ & $4(44 \%)$ & 0.470 \\
\hline Psychiatric & $2(9 \%)$ & $0(0 \%)$ & 0.240 & $2(10 \%)$ & $0(0 \%)$ & 0.334 \\
\hline Gastroenteric & $4(17 \%)$ & $6(40 \%)$ & 0.122 & $2(10 \%)$ & $3(33 \%)$ & 0.135 \\
\hline COPD & $8(35 \%)$ & $1(7 \%)$ & 0.046 & $7(35 \%)$ & $1(11 \%)$ & 0.188 \\
\hline Autoimmune & $3(13 \%)$ & $2(13 \%)$ & 0.979 & $3(15 \%)$ & $1(11 \%)$ & 0.776 \\
\hline Nephrologic & $6(26 \%)$ & $6(40 \%)$ & 0.367 & $3(15 \%)$ & $4(44 \%)$ & 0.096 \\
\hline Neoplastic & $2(9 \%)$ & $1(7 \%)$ & 0.820 & $2(10 \%)$ & $1(11 \%)$ & 0.936 \\
\hline Metabolic & $10(43 \%)$ & $3(20 \%)$ & 0.136 & $9(45 \%)$ & $2(22 \%)$ & 0.245 \\
\hline Genetic & $1(4 \%)$ & $2(13 \%)$ & 0.315 & $1(5 \%)$ & $1(11 \%)$ & 0.561 \\
\hline Pregnancy & $0(0 \%)$ & $1(7 \%)$ & 0.209 & $0(0 \%)$ & $1(11 \%)$ & 0.138 \\
\hline Diabetes & $6(26 \%)$ & $3(20 \%)$ & 0.666 & $5(25 \%)$ & $2(22 \%)$ & 0.864 \\
\hline Charlson comorbidity index & $5(2-7)$ & $4(2-5)$ & 0.209 & $5(2-7)$ & $4(2-5)$ & 0.209 \\
\hline \multicolumn{7}{|l|}{ Influenza type } \\
\hline A & $13(57 \%)$ & $12(80 \%)$ & 0.136 & $8(40 \%)$ & $6(80 \%)$ & 0.050 \\
\hline $\mathrm{A}-\mathrm{H} 1 \mathrm{~N} 1$ & $10(43 \%)$ & $3(20 \%)$ & 0.135 & $3(15 \%)$ & $3(33 \%)$ & 0.276 \\
\hline B & $0(0 \%)$ & $0(0 \%)$ & 1.000 & $0(0 \%)$ & $0(0 \%)$ & 1.000 \\
\hline \multicolumn{7}{|l|}{ Severity } \\
\hline ICU & $12(52 \%)$ & $0(0 \%)$ & 0.002 & $5(25 \%)$ & $0(0 \%)$ & 0.105 \\
\hline SOFA score & $3(2-4)$ & $1(1-2)$ & 0.004 & $3(2-4)$ & $1(1-2)$ & 0.013 \\
\hline No oxygen support & $1(4 \%)$ & $3(20 \%)$ & 0.124 & $1(5 \%)$ & $7(78 \%)$ & $\begin{array}{l}< \\
0.001\end{array}$ \\
\hline NIV & $4(17 \%)$ & $1(7 \%)$ & 0.339 & $4(20 \%)$ & $1(11 \%)$ & 0.559 \\
\hline IMV & $8(35 \%)$ & $0(0 \%)$ & 0.031 & $7(35 \%)$ & $0(0 \%)$ & 0.045 \\
\hline ECMO & $2(9 \%)$ & $0(0 \%)$ & 0.240 & $2(10 \%)$ & $0(0 \%)$ & 0.334 \\
\hline \multicolumn{7}{|l|}{ Pulmonary superinfection } \\
\hline Total superinfection & $7(30 \%)$ & $0(0 \%)$ & 0.021 & $6(30 \%)$ & $0(0 \%)$ & 0.069 \\
\hline A. baumannii, P. jiroveci & $1(4 \%)$ & $0(0 \%)$ & 0.413 & $1(5 \%)$ & $0(0 \%)$ & 0.502 \\
\hline A. baumannii & $1(4 \%)$ & $0(0 \%)$ & 0.413 & $1(5 \%)$ & $0(0 \%)$ & 0.502 \\
\hline $\begin{array}{l}\text { MRSA, S. maltophila, A. } \\
\text { fumigatus }\end{array}$ & $1(4 \%)$ & $0(0 \%)$ & 0.413 & $1(5 \%)$ & $0(0 \%)$ & 0.502 \\
\hline C. striatum & $1(4 \%)$ & $0(0 \%)$ & 0.413 & $1(5 \%)$ & $0(0 \%)$ & 0.502 \\
\hline A. fumigatus & $3(13 \%)$ & $0(0 \%)$ & 0.413 & $2(10 \%)$ & $0(0 \%)$ & 0.334 \\
\hline
\end{tabular}

Outcome 
Table 1 Baseline characteristics, severity, and microbiology of pulmonary superinfections and outcomes (Continued)

\begin{tabular}{|c|c|c|c|c|c|c|}
\hline \multirow[t]{2}{*}{ Characteristics } & \multicolumn{3}{|c|}{ Total influenza pneumonia (38) } & \multicolumn{3}{|l|}{ Influenza CAP (29) } \\
\hline & $\begin{array}{l}<724 \text { lymphocytes } / \mu \text { l } \\
(n=23)\end{array}$ & $\begin{array}{l}>724 \text { lymphocytes } / \mu \text { l } \\
(n=15)\end{array}$ & $\begin{array}{l}p \\
\text { value }^{*}\end{array}$ & $\begin{array}{l}<724 \text { lymphocytes } / \mu \text { l } \\
(n=20)\end{array}$ & $\begin{array}{l}\text { > } 724 \text { lymphocytes } / \mu \text { l } \\
(n=9)\end{array}$ & $\begin{array}{l}p \\
\text { value }\end{array}$ \\
\hline LOS, days & $20(11-40)$ & $22(11-45)$ & 0.633 & $20(11-39)$ & $12(10-22)$ & 0.633 \\
\hline Mortality & $7(30 \%)$ & $2(13 \%)$ & 0.411 & $6(30 \%)$ & $1(11 \%)$ & 0.277 \\
\hline
\end{tabular}

Data are presented as median (interquartile range (IQR) 25-75\%) for continuous variables or as simple frequencies ( $n$ ) and percentages for categorical variables $N / L$ ratio neutrophils/lymphocytes ratio, CAP community-acquired pneumonia, HAP hospital-acquired pneumonia, COPD chronic obstructive pulmonary disease, $I C U$ intensive care unit, SOFA score Sequential Organ Failure Assessment score, NIV non-invasive ventilation, IMV invasive mechanical ventilation, ECMO extracorporeal membrane oxygenation, LOS length of stay

*For comparisons between groups, Fisher's exact test was used for dichotomous variables, the $x^{2}$ test was used for non-ordered categorical variables and the Mann-Whitney test was used for continuous variables

\section{Acknowledgements}

None

\section{Authors' contributions}

VB and GdE designed and drafted the study. VB and LC sorted the data. CB, GC, and GdE analyzed the data. MV, GdE, and GC contributed substantially to its revision. MV takes responsibility for the paper as a whole. All authors read and approved the final manuscript.

\section{Funding}

None

Availability of data and materials

All data are available on request from the corresponding author.

\section{Ethics approval and consent to participate}

Approved by the Institutional Board of Public Health and Infectious Diseases of Sapienza University.

\section{Consent for publication}

All authors sign a consensus for participating.

\section{Competing interests}

The authors declare that they have no competing interests.

Received: 14 August 2019 Accepted: 13 September 2019

Published online: 29 October 2019

\section{References}

1. Méndez R, Menéndez R, Amara-Elori I, Feced L, Piró A, Ramírez P, Sempere A, Ortega A, Bermejo-Martín JF, Torres A. Lymphopenic communityacquired pneumonia is associated with a dysregulated immune response and increased severity and mortality. J Inf Secur. 2019;78(6):423-31.

2. Bermejo-Martin JF, Cilloniz C, Mendez R, Almansa R, Gabarrus A, Ceccato A, Torres A, Menendez R, NEUMONAC group. Lymphopenic community acquired pneumonia (L-CAP), an immunological phenotype associated with higher risk of mortality. EBioMedicine. 2017;24:231-6.

3. Zhou F, Li H, Gu L, Liu M, Xue C, Cao B, Wang C, for the National Influenza A(H1N1)pdm09 Clinical Investigation Group of China. Risk factors for nosocomial infection among hospitalised severe influenza A(H1N1)pdm09 patients. Respir Med. 2018;134:86-91.

4. Giannella M, Rodríguez-Sánchez B, Roa PL, Catalán P, Muñoz P, García de Viedma D, Bouza E, Gregorio Marañón Task Force for Pneumonia GANG. Should lower respiratory tract secretions from intensive care patients be systematically screened for influenza virus during the influenza season? Crit Care. 2012;16(3):R104.

5. Chongnarungsin D, Yella J, Guzman J. Coinfection of h1n1 influenza with Pneumocystis jiroveci pneumonia in immunocompetent patient. Crit Care Med. 2014;42(12):1634

\section{Publisher's Note}

Springer Nature remains neutral with regard to jurisdictional claims in published maps and institutional affiliations. 